\title{
Violation of the Spatial Quantum Inequality and the Quantum Interest Conjecture: A New Approach
}

\author{
Dan Solomon \\ Rauland-Borg Corporation \\ 1802 West Central Road, Mount Prospect, IL 60056, USA \\ E-mail: dan.solomon@rauland.com
}

Received: September 22, 2011 Accepted: October 12, 2011 Published: February 1, 2012

doi:10.5539/apr.v4n1p109 URL: http://dx.doi.org/10.5539/apr.v4n1p109

\begin{abstract}
It is well known that the local kinetic energy density can be negative in quantum field theory. This is a "quantum effect" that is in contrast to classical physics where the kinetic energy density is always non-negative. It has been proposed in the literature that there exist "quantum inequalities" which place limits on this negative energy density. In this paper we will examine a type of quantum inequality call the spatial quantum inequality. The spatial quantum inequality is a lower bound on the weighted average of the kinetic energy density. It will be shown that for a massless scalar field in 1-1 dimensional space-time we can formulate a quantum state which violates the quantum inequality. In addition, we will look into the quantum interest conjecture. The quantum interest conjecture states that a negative energy pulse must always be closely associated with a positive energy pulse of greater magnitude. It will be shown that the quantum interest conjecture is violated.
\end{abstract}

Keywords: Spatial quantum inequality, Quantum interest conjecture

\section{Introduction}

In quantum field theory the kinetic energy density can be negative over some region of space (Epstein et al., 1965). This is a "quantum effect" that is in contrast to classical physics where the kinetic energy density is always positive. One possible consequence of this is that the existence of negative energy could give rise to exotic phenomenon such as traversable wormholes (Morris et al., 1988) and violations of the second law of thermodynamics (Ford, 1978).

There are a number of papers that claim to show that there are limits on this negative energy density (Ford et al., 1998; Flanagan, 1997; Fewster et al., 1998; Ford, 2010; Bostelann et al., 2009; Fewster et al., 2008; Eveson et al., 2007; Fewster, 2005; Ford et al., 1998; Pfenning et al., 1998). These limits are called the quantum inequalities. The quantum inequalities provide a lower bound on the weighted average of the kinetic energy density integrated over some region of space and time. They apply to "free field" systems, that is, systems where all external potentials are zero. It is argued that the presence of the quantum inequalities eliminates the possibility of the exotic phenomenon mentioned above.

The spatial quantum inequality will be the focus of the discussion of this paper. The spatial inequality is the proposition that there is a lower bound to the weighted average of the kinetic energy density integrated over some region of space at a given point in time. It has been claimed to apply to a massless scalar field in 1-1 dimensional space-time (Flanagan, 1997).

I have proposed in a number of papers that one can formulate counterexamples to the quantum inequalities (Solomon, 2010; 2011A; 2011B; 2011C). The basis of these counterexamples will be described as follows: Consider a massless scalar field in 1-1 dimensional space-time and assume that all external potentials are zero. Next consider some finite region in space $a>x>-a$. Call this region $I$. Region $I I$ will be a region outside of region $I$ consisting of $R>x>a$ and $-a>x>-R$. If the kinetic energy density is zero in region $I I$ and if the average kinetic energy density is negative inside region $I$ then the spatial quantum inequality will be violated if $R$ is sufficiently large.

Basically the spatial quantum inequality will be violated if a "free field" quantum state exists which includes an "isolated" region of negative kinetic energy density. By "isolated" we mean that the region of negative kinetic energy density is a sufficiently large distance away from any positive kinetic energy density that may exist. In 
this paper we will demonstrate the existence of a "free field" quantum system for which a region of negative kinetic energy density is "isolated" and violates the spatial quantum inequality.

\section{Spatial Quantum Inequality}

In this section we will show that a system that contains an isolated region of negative kinetic energy density can violate the spatial quantum inequality. According to Flanagen (1997) the spatial quantum inequality is expressed by the following equation,

$$
\int_{-\infty}^{+\infty} T_{00 R}(x, t) \rho(x) d x \geq \xi_{S, \min }[\rho]
$$

where $T_{00 R}(x, t)$ is the renormalized kinetic energy density and $\rho(x)$ is a positive weighting function which satisfies,

$$
\int_{-\infty}^{+\infty} \rho(x) d x=1
$$

and,

$$
\xi_{S, \min }[\rho]=-\frac{1}{24 \pi} \int_{-\infty}^{+\infty} d x \frac{\rho^{\prime}(x)^{2}}{\rho(x)}
$$

where $\rho^{\prime}(x)=d \rho(x) / d x$. It will now be shown that an isolated region of negative kinetic energy density violates the spatial quantum inequality. Define the weighting factor $\rho(x)$ according to,

$$
\rho(x)=N \times\left\langle\begin{array}{c}
1 \text { for }|x|<a \\
\cos ^{2}[f(|x|-a)] \text { for } R>|x|>a \\
0 \text { for }|x|>R
\end{array}\right.
$$

where $N=1 /(R+a)$ and $f=(\pi / 2) /(R-a)$. Note that $\rho(x)$ has finite support which is allowed according to Flanagan (1997). Use this in (2.3) to obtain,

$$
\xi_{S, \min }=-\frac{1}{6}\left(\frac{1}{\left(R^{2}-a^{2}\right)}\right)
$$

Assume that the kinetic energy density is zero in the region $R>|x|>a$ and the average value of the kinetic energy density in the region $|x|<a$ is given by $T_{00 R, a v e}=-\eta$ where $\eta>0$. For this situation the negative energy density is isolated. That is, the region of negative kinetic energy density is separated from any positive kinetic energy density that may exist by a minimum distance of $R-a$. Use this along with (2.4) to obtain,

$$
\int_{-\infty}^{+\infty} T_{00 R}(x, t) \rho(x) d x=\frac{-2 a \eta}{(R+a)}
$$

If the spatial quantum inequality holds then Eq. (2.1) must be valid which implies,

$$
\frac{-2 a \eta}{(R+a)}>-\frac{1}{6}\left(\frac{1}{\left(R^{2}-a^{2}\right)}\right)
$$

This in turn yields, 


$$
\frac{2 a \eta}{(R+a)}<\frac{1}{6}\left(\frac{1}{\left(R^{2}-a^{2}\right)}\right)
$$

For sufficiently large $R$ this equation will not be true. Therefore the spatial quantum inequality can fail if we can formulate a system which contains an isolated region of negative kinetic energy density.

\section{Isolated Negative Kinetic Energy Density}

In this section we will examine the kinetic energy density for a massless scalar field in 1-1 dimensional space-time with the goal of trying to demonstrate the existence of a quantum state for which includes a region of negative kinetic energy density which is isolated. This is easy to do if we allow for the presence of a non-zero scalar potential.

It can be shown that one can produce an isolated region of negative kinetic energy density for scalar fields in the presence of an external scalar potential. For example, Mamaev et al. (1981) considered a massless scalar field in 1-1 dimensions in the presence of the scalar potential given by,

$$
V_{\lambda}(x)=\lambda[\delta(x-a / 2)+\delta(x+a / 2)]
$$

where $\lambda$ is a non-negative constant which can be set equal to zero to turn the potential off (Also see Solomon, 2011A; Mostepanenko et al., 1997; Mamaev et al., 1981) show that for this system the renormalized kinetic energy density, $T_{00 R}(x)$, is given by,

$$
T_{00 R}(x)=\left\{\begin{array}{c}
-\eta \text { if }|x|<a / 2 \\
0 \text { if }|x|>a / 2
\end{array}\right.
$$

where $\eta$ is a positive constant. Therefore $T_{00 R}(x)$ is negative in the region between $-a / 2$ and $+a / 2$ and zero elsewhere.

Another example is given in Solomon (2011B) which derived an expression for the kinetic energy density of a quantum state in the presence of a static scalar potential $\lambda V_{0}(x)$ where $V_{0}(x)$ is an inverse square well potential given by,

$$
V_{0}(x)=\left\{\begin{array}{c}
V_{0} \text { for }|x|<a \\
0 \text { for }|x|>a
\end{array}\right.
$$

where $V_{0}$ is a positive constant and $\lambda$ is a positive constant that can be set to zero to turn off the potential. It was shown for this situation that $T_{00 R}(x)=0$ for $|x|>a$ and that in the region $|x|<a$ the average value of $T_{00 R}(x)$ is negative.

It is evident in both these examples that the negative part of the kinetic energy density is isolated. This would be a violation of the spatial quantum inequality except for the presence of a non-zero scalar potential. This is due to the fact that the spatial quantum inequality only applies to the "free field" case where the scalar potential is zero. This problem will be dealt with in Section 5. Meanwhile, in this rest of this section, we will briefly review the discussion in Solomon (2011B) where the kinetic energy density was determined for the massless scalar field in the presence of the scalar potential given by (3.3).

We will operate in the Schrödinger picture. In this case the field operators $\hat{\varphi}(x)$ and $\hat{\pi}(x)$ are time independent and obey the commutation relationships,

$$
[\hat{\varphi}(y), \hat{\pi}(x)]=i \delta(x-y)
$$

with all other commutations equal to zero. The kinetic energy density operator is given by,

$$
\hat{T}_{00}(x)=\frac{1}{2}\left(\hat{\pi}(x) \cdot \hat{\pi}(x)+\frac{d \hat{\varphi}(x)}{d x} \cdot \frac{d \hat{\varphi}(x)}{d x}\right)
$$


The field operators are given by,

$$
\begin{gathered}
\hat{\varphi}(x)=\int_{0}^{\infty} \frac{d k}{\sqrt{2 \pi|k|}} \sum_{\chi=+,-}\left(\hat{a}_{\lambda, k}^{\chi}+\hat{a}_{\lambda, k}^{\chi^{*}}\right) \psi_{\lambda, k}^{\chi}(x) \\
\hat{\pi}(x)=i \int_{0}^{\infty} \frac{|k| d k}{\sqrt{2 \pi|k|}} \sum_{\chi=+,-}\left(\hat{a}_{\lambda, k}^{\chi^{*}}-\hat{a}_{\lambda, k}^{\chi}\right) \psi_{\lambda, k}^{\chi}(x)
\end{gathered}
$$

where the $\psi_{\lambda, k}^{\chi}(x)$ are real with $\psi_{\lambda, k}^{+}(x)$ representing the symmetric solutions and $\psi_{\lambda, k}^{-}(x)$ representing the anti-symmetric solutions. These are given in Solomon (2011D). The $\hat{a}_{\lambda, k}^{\chi^{*}}$ and $\hat{a}_{\lambda, k}^{\chi}$ are the creation and destruction operators, respectively. They obey the commutation relationships $\left[\hat{a}_{\lambda, k^{\prime}}^{\chi^{\prime}}, \hat{a}_{\lambda, k}^{\chi^{*}}\right]=\delta_{\chi^{\prime} \chi} \delta\left(k^{\prime}-k\right)$ with all other commutations being zero. The $\psi_{\lambda, k}^{\chi}(x)$ are solutions of,

$$
-k^{2} \psi_{\lambda, k}^{\chi}(x)-\frac{d^{2} \psi_{\lambda, k}^{\chi}(x)}{d x^{2}}+\lambda V_{0}(x) \psi_{\lambda, k}^{\chi}(x)=0
$$

where,

$$
\int_{-\infty}^{+\infty} d x \psi_{\lambda, k}^{+}(x) \psi_{\lambda, k^{\prime}}^{-}(x)=0 \text { and } \sum_{\chi=+,-} \int_{-\infty}^{+\infty} \psi_{\lambda, k}^{\chi}(x) \psi_{\lambda, k^{\prime}}^{\chi}(x) d x=2 \pi \delta\left(k-k^{\prime}\right)
$$

The state $\left|0_{\lambda}\right\rangle$ is defined by,

$$
\hat{a}_{\lambda, k}^{\chi}\left|0_{\lambda}\right\rangle=0 \text { and }\left\langle 0_{\lambda} \mid 0_{\lambda}\right\rangle=1
$$

The "unrenormalized" kinetic energy density expectation value of the state $\left|0_{\lambda}\right\rangle$ is given by,

$$
T_{00}\left(x ;\left|0_{\lambda}\right\rangle\right)=\left\langle 0_{\lambda}\left|\hat{T}_{00}(x)\right| 0_{\lambda}\right\rangle
$$

Use (3.6), (3.7), and (3.10) in the above to obtain,

$$
T_{00}\left(x ;\left|0_{\lambda}\right\rangle\right)=\int_{0}^{\infty}\left(\frac{d k}{2 \pi|k|}\right) \sum_{\chi=+,-} \frac{1}{2}\left[k^{2} \psi_{\lambda, k}^{\chi}(x)^{2}+\left(\frac{d \psi_{\lambda, k}^{\chi}(x)}{d x}\right)^{2}\right] d k
$$

The problem with this result is that it is infinite. In order to "regularize" this quantity we proceed as in Graham et al. (2003) and introduce a counter term to compensate for the cosmological constant. This involves subtracting off the term,

$$
T_{00}\left(x ;\left|0_{0}\right\rangle\right)=\int_{0}^{\infty}\left(\frac{d k}{2 \pi|k|}\right) \sum_{\chi=+,-} \frac{1}{2}\left[k^{2} \psi_{0, k}^{\chi}(x)^{2}+\left(\frac{d \psi_{0, k}^{\chi}(x)}{d x}\right)^{2}\right] d k
$$

where,

$$
\psi_{0, k}^{+}(x)=\cos (k x) \text { and } \psi_{0, k}^{-}(x)=\sin (k x)
$$

Note that $T_{00}\left(x ;\left|0_{0}\right\rangle\right)$ is derived from $T_{00, \lambda}\left(x ;\left|0_{\lambda}\right\rangle\right)$ by setting $\lambda=0 . T_{00}\left(x ;\left|0_{0}\right\rangle\right)$ may be thought of as the unregularized kinetic energy density when the scalar potential is zero.

The regularized kinetic energy density is, then, 


$$
T_{00 R}\left(x ;\left|0_{\lambda}\right\rangle\right)=\int_{0}^{\infty}\left(\frac{d k}{4 \pi|k|}\right) \sum_{\chi=+,-}\left[k^{2}\left(\psi_{\lambda, k}^{\chi}(x)^{2}-\psi_{0, k}^{\chi}(x)^{2}\right)+\left(\frac{d \psi_{\lambda, k}^{\chi}(x)}{d x}\right)^{2}-\left(\frac{d \psi_{0, k}^{\chi}(x)}{d x}\right)^{2}\right]
$$

Referring to Solomon (2011B) we find that in the region $|x|>a$,

$$
T_{00 R}\left(x ;\left|0_{\lambda}\right\rangle\right) \underset{|x|>a}{=0}
$$

As for the region $|x|<a$ define,

$$
E_{K E}=\int_{-a}^{+a} T_{00 R}\left(x ;\left|0_{\lambda}\right\rangle\right) d x
$$

From Solomon (2011B) it is shown that,

$$
E_{K E}=-\frac{V_{0}^{2}}{4 \pi} \int_{0}^{\infty} d \kappa\left\{\frac{2 a \kappa^{\prime} \cosh \left(2 a \kappa^{\prime}\right)-\sinh \left(2 a \kappa^{\prime}\right)}{\kappa^{\prime 2} D_{\kappa}}\right\}
$$

where we have set $\lambda=1$ and,

$$
D_{\kappa}=2 \kappa^{\prime} \kappa \cosh \left(2 \kappa^{\prime} a\right)+\left(\kappa^{2}+\kappa^{\prime 2}\right) \sinh \left(2 \kappa^{\prime} a\right)
$$

where $\kappa^{\prime}=\sqrt{\kappa^{2}+V_{0}}$. The integrand in (3.18) is non-negative for all $\kappa \geq 0$. Therefore $E_{K E}<0$. The result of this is that the total kinetic energy is negative in the interval $-a<x<a$ and the kinetic energy density is zero outside of this region.

Therefore the average energy density in the region $-a<x<a$ is,

$$
T_{00 R, A v e}\left(\left|0_{\lambda}\right\rangle\right)=-\frac{\left|E_{K E}\right|}{2 a} \text { for }-a<x<a
$$

where we have used $E_{K E}=-\left|E_{K E}\right|$ since $E_{K E}$ is negative. This is done to emphasis the fact that $T_{00 R, A v e}\left(\left|0_{\lambda}\right\rangle\right)$ is negative.

\section{The Translation Operator}

At this point we have demonstrated the existence of a quantum state for which the negative part of the kinetic energy density is isolated. This would be a violation of the spatial quantum inequality except for the fact that this quantum state exists in the presence of a non-zero scalar potential given by (3.3). For the quantum inequalities to apply the scalar potential must be removed.

In two papers by the author (Solomon, 2011A; Solomon, 2011B) it was argued that the kinetic energy density was continuous with respect to a sudden change of the scalar potential. If this is true then the scalar potential could be instantaneously set to zero without producing an instantaneous change in the kinetic energy density. Using this procedure it was shown that we can produce a free field system in which the kinetic energy density violates the spatial quantum inequality.

In this paper a somewhat different approach will be used. Consider the kinetic energy density $T_{00 R}\left(x ;\left|0_{\lambda}\right\rangle\right)$ determined in the last section. As was shown above this will be zero in the region $|x|>a$ and its average value will be negative in the region $|x|<a$. Next assume that there exists a spatial translation operator $\hat{P}_{L}$ and consider the state vector $\left|\Omega_{0}\right\rangle=\hat{P}_{L}\left|0_{\lambda}\right\rangle$. It will be shown that the renormalized kinetic energy density of the state $\left|\Omega_{0}\right\rangle$ is,

$$
T_{00 R}\left(x ;\left|\Omega_{0}\right\rangle\right)=T_{00 R}\left(x+L ;\left|0_{\lambda}\right\rangle\right)
$$


Therefore the kinetic energy density of the state $\left|\Omega_{0}\right\rangle$ is equal to the kinetic energy density of the state $\left|0_{\lambda}\right\rangle$ shifted by the distance $L$ in the negative x-direction. The state $\left|\Omega_{0}\right\rangle$ will therefore contain an isolated region of negative kinetic energy density in the region $-a-L<x<a-L$. The kinetic energy density outside of this region is zero. For $L \rightarrow \infty$ this region of negative energy density will be an infinite distance away from the non-zero scalar potential located in the region $|x|<a$. Therefore the sudden removable of this scalar potential cannot effect the negative energy density region associated with the state $\left|\Omega_{0}\right\rangle$ because it an infinite distance away. This fact will be used to show that the state $\left|\Omega_{0}\right\rangle$ violates the spatial quantum inequality.

In the following discussion we will specify the translation operator $\hat{P}_{L}$. Start by defining the operator,

$$
\hat{C}=\frac{1}{2} \int\left(\hat{\pi}(x) \frac{\partial \hat{\varphi}(x)}{\partial x}+\frac{\partial \hat{\varphi}(x)}{\partial x} \hat{\pi}(x)\right) d x
$$

Using the commutation relationships (3.4) we obtain,

$$
[\hat{\varphi}(x), \hat{C}]=i \frac{\partial \hat{\varphi}(x)}{\partial x} \text { and }[\hat{\pi}(x), \hat{C}]=i \frac{\partial \hat{\pi}(x)}{\partial x}
$$

The translation operator will be given by,

$$
\hat{P}_{L}=e^{-i \hat{C} L}
$$

Use the fact that $\hat{C}^{\dagger}=\hat{C}$ to obtain,

$$
\hat{P}_{L}^{\dagger}=e^{+i \hat{C} L}
$$

Next use the Baker-Campell-Hausdorff (Greiner et al., 1992) relationships to obtain,

$$
\hat{P}_{L}^{\dagger} \hat{\varphi}(x) \hat{P}_{L}=\hat{\varphi}(x)-i L[\hat{\varphi}(x), \hat{C}]+\frac{(-i)^{2} L^{2}}{2}[[\hat{\varphi}(x), \hat{C}], \hat{C}]+\ldots
$$

Use this and (4.3) to obtain,

$$
\hat{P}_{L}^{\dagger} \hat{\varphi}(x) \hat{P}_{L}=\hat{\varphi}(x)+\frac{\partial \hat{\varphi}(x)}{\partial x} L+\frac{1}{2} \frac{\partial^{2} \hat{\varphi}(x)}{\partial x^{2}} L^{2}+\ldots=\hat{\varphi}(x+L)
$$

where the last step is based on the Taylor's expansion of the quantity $\hat{\varphi}(x+L)$. Similarly we can show that,

$$
\hat{P}_{L}^{\dagger} \hat{\pi}(x) \hat{P}_{L}=\hat{\pi}(x+L)
$$

From the above we obtain,

$$
\hat{P}_{L}^{\dagger} \hat{T}_{00}(x) \hat{P}_{L}=\hat{T}_{00}(x+L)
$$

where we have used $\hat{P}_{L}^{\dagger} \hat{P}_{L}=1$.

Next, define the state,

$$
\left|\Omega_{0}\right\rangle=\hat{P}_{L}\left|0_{\lambda}\right\rangle
$$

The unrenormalized kinetic energy density of this state is,

$$
T_{00}\left(x ;\left|\Omega_{0}\right\rangle\right)=\left\langle\Omega_{0}\left|\hat{T}_{00}(x)\right| \Omega_{0}\right\rangle=\left\langle 0_{\lambda}\left|\hat{P}_{L}^{\dagger} \hat{T}_{00}(x) \hat{P}_{L}\right| 0_{\lambda}\right\rangle=\left\langle 0_{\lambda}\left|\hat{T}_{00}(x+L)\right| 0_{\lambda}\right\rangle
$$

This allows us to write, 


$$
T_{00}\left(x ;\left|\Omega_{0}\right\rangle\right)=T_{00}\left(x+L ;\left|0_{\lambda}\right\rangle\right)
$$

Using the regularization procedure of Section 3 we obtain the renormalized kinetic energy density of the state $\left|\Omega_{0}\right\rangle$ as,

$$
T_{00 R}\left(x ;\left|\Omega_{0}\right\rangle\right)=T_{00 R}\left(x+L ;\left|0_{\lambda}\right\rangle\right)
$$

Therefore by using the results from the end of Section 3 we obtain,

$$
T_{00 R}\left(x ;\left|\Omega_{0}\right\rangle\right)=0 \text { for }|x+L|>a
$$

and, in the region $|x+L|<a$, the average value of $T_{00 R}\left(x ;\left|\Omega_{0}\right\rangle\right)$ is,

$$
T_{00 R, A v e}\left(\left|\Omega_{0}\right\rangle\right)=-\frac{\left|E_{K E}\right|}{2 a} \text { for }|x+L|<a
$$

\section{Removing the Scalar Potential}

In the last section we defined the state $\left|\Omega_{0}\right\rangle=\hat{P}_{L}\left|0_{\lambda}\right\rangle$. The kinetic energy of this state would be considered as violating the spatial quantum inequality if the scalar potential was zero. Therefore we want to determine what happens to the kinetic energy density of the state $\left|\Omega_{0}\right\rangle$ when we remove the scalar potential $\lambda V_{0}(x)$.

In order to do this we have to consider the time evolution of the system as the scalar potential is removed. We start by defining the initial state of the system at time $t=0$. At this point in time the scalar potential is the static potential $\lambda V_{0}(x)$ defined in (3.3). This allows us to specify the state $\left|0_{\lambda}\right\rangle$. Using the translation operator we can then specify the state $\left|\Omega_{0}\right\rangle=\hat{P}_{L}\left|0_{\lambda}\right\rangle$. The kinetic energy density of the state $\left|\Omega_{0}\right\rangle$ is the same as the kinetic energy density of the state $\left|0_{\lambda}\right\rangle$ except that it is shifted in the negative $x$-direction by the amount $L$.

Next let $L \rightarrow \infty$ and divide up space into two regions. Region A consists of all points for which $x<-L / 2$ and region $\mathrm{B}$ consists of all points where $-L / 2<x$. The inverse square well potential is located in region $\mathrm{B}$ per Eq. (3.3). For the state $\left|\Omega_{0}\right\rangle$ the negative part of the kinetic energy density is located in region A per (4.15). Next we want to determine how the kinetic energy density of the state $\left|\Omega_{0}\right\rangle$ changes as we remove the scalar potential. Assume that the scalar potential is reduced to zero during the time interval $0<t<t_{1}$ where $L / 2>>t_{1}$. To make this happen let the scalar potential be given by $\lambda(t) V_{0}(x)$ where,

$$
\lambda(t)=\left\{\begin{array}{c}
1 \text { for } t<0 \\
1-\left(t / t_{1}\right) \text { for } 0<t<t_{1} \\
0 \text { for } t \geq t_{1}
\end{array}\right.
$$

Now the removal of the potential in Region B cannot possibly effect any changes in the kinetic energy density in Region A because any disturbance caused by the changing potential cannot travel faster than the speed of light. The kinetic energy density in region A evolves in time independently of the removal of the potential for all time $t$ such that $L / 2>t$. Therefore the details of the removal of the potential will not impact the kinetic energy density in region A. The negative kinetic energy density, which at time $t=0$ is confined to the region $|x+L|<a$, will simply spread out from this region at the speed of light during the interval from 0 to $t_{1}$. At $t_{1}$ the negative energy density in region A will be confined to the region $|x+L|<a+t_{1}$. The result will be a region of negative kinetic energy density surrounded by an arbitrarily large region where the kinetic energy density is zero. As was shown in the Section 2 this violates the spatial quantum inequality. The above analysis will be proven in the following discussion.

The time evolution of the quantum state $\left|\Omega_{0}\right\rangle=\hat{P}_{L}\left|0_{\lambda}\right\rangle$ for $t>0$ is given by the Schrödinger equation, 


$$
i \frac{\partial\left|\Omega_{0}(t)\right\rangle}{\partial t}=\left(\hat{H}_{0}+\lambda(t) \hat{V}_{0}(x)\right)\left|\Omega_{0}(t)\right\rangle
$$

The dual equation is,

$$
-i \frac{\partial\left\langle\Omega_{0}(t)\right|}{\partial t}=\left\langle\Omega_{0}(t)\right|\left(\hat{H}_{0}+\lambda(t) \hat{V}_{0}(x)\right)
$$

where $\hat{H}_{0}$ is the free field Hamiltonian operator and is given by,

$$
\hat{H}_{0}=\int \hat{T}_{00}(x) d x
$$

and,

The initial condition at $t=0$ is,

$$
\hat{V}_{0}(x)=\frac{1}{2} \int V_{0}(x) \hat{\varphi}(x) \cdot \hat{\varphi}(x) d x
$$

$$
\left|\Omega_{0}(0)\right\rangle=\left|\Omega_{0}\right\rangle=\hat{P}_{L}\left|0_{\lambda}\right\rangle
$$

Use the commutation relationships (3.4) to obtain,

$$
\left[\hat{H}_{0}, \hat{T}_{00}(x)\right]=-i \frac{\partial \hat{T}_{10}(x)}{\partial x}
$$

where $\hat{T}_{10}(x)$ is defined by,

$$
\hat{T}_{10}(x)=\frac{1}{2}\left(\hat{\pi}(x) \frac{\partial \hat{\varphi}(x)}{\partial x}+\frac{\partial \hat{\varphi}(x)}{\partial x} \hat{\pi}(x)\right)
$$

Use (3.6) and (3.7) in the above to obtain,

$$
T_{10}\left(x ;\left|0_{\lambda}\right\rangle\right)=\left\langle 0_{\lambda}\left|\hat{T}_{10}(x)\right| 0_{\lambda}\right\rangle=0
$$

Note that $\left\langle 0_{\lambda}\left|\hat{T}_{10}(x)\right| 0_{\lambda}\right\rangle$ does not require a regularization step. It is finite as defined.

Using the commutation relationships along with (5.2) and (5.3) we obtain,

$$
\begin{aligned}
\frac{\partial}{\partial t}\left\langle\Omega_{0}(t)\left|\hat{T}_{00}(x)\right| \Omega_{0}(t)\right\rangle= & \frac{\partial}{\partial x}\left\langle\Omega_{0}(t)\left|\hat{T}_{10}(x)\right| \Omega_{0}(t)\right\rangle \\
& -\frac{1}{2} \lambda(t) V_{0}(x) \frac{\partial}{\partial t}\left\langle\Omega_{0}(t)|\hat{\varphi}(x) \cdot \hat{\varphi}(x)| \Omega_{0}(t)\right\rangle
\end{aligned}
$$

and,

$$
\begin{aligned}
\frac{\partial}{\partial t}\left\langle\Omega_{0}(t)\left|\hat{T}_{10}(x)\right| \Omega_{0}(t)\right\rangle= & \frac{\partial}{\partial x}\left\langle\Omega_{0}(t)\left|\hat{T}_{00}(x)\right| \Omega_{0}(t)\right\rangle \\
& -\frac{1}{2} \lambda(t) V_{0}(x) \frac{\partial}{\partial x}\left\langle\Omega_{0}(t)|\hat{\varphi}(x) \cdot \hat{\varphi}(x)| \Omega_{0}(t)\right\rangle
\end{aligned}
$$

Now consider the quantity $\left\langle\Omega_{0}(t)\left|\hat{T}_{00}(x)\right| \Omega_{0}(t)\right\rangle$ in the above equations. Technically this quantity is infinite and undefined. The process of renormalizing this quantity involves subtracting off an infinite constant as discussed in Section 3. Since the derivative of this infinite constant is zero we can write, 
Similarly,

$$
\frac{\partial}{\partial t} T_{00 R}\left(x ;\left|\Omega_{0}(t)\right\rangle\right)=\frac{\partial}{\partial t}\left\langle\Omega_{0}(t)\left|\hat{T}_{00}(x)\right| \Omega_{0}(t)\right\rangle
$$

Also define,

$$
\frac{\partial}{\partial x} T_{00 R}\left(x ;\left|\Omega_{0}(t)\right\rangle\right)=\frac{\partial}{\partial x}\left\langle\Omega_{0}(t)\left|\hat{T}_{00}(x)\right| \Omega_{0}(t)\right\rangle
$$

$$
T_{10}\left(x ;\left|\Omega_{0}(t)\right\rangle\right)=\left\langle\Omega_{0}(t)\left|\hat{T}_{10}(x)\right| \Omega_{0}(t)\right\rangle
$$

Use the above to obtain,

$$
\frac{\partial T_{00 R}\left(x ;\left|\Omega_{0}(t)\right\rangle\right)}{\partial t}=\frac{\partial T_{10}\left(x ;\left|\Omega_{0}(t)\right\rangle\right)}{\partial x}-\frac{1}{2} \lambda(t) V_{0}(x) \frac{\partial}{\partial t}\left\langle\Omega_{0}(t)|\hat{\varphi}(x) \cdot \hat{\varphi}(x)| \Omega_{0}(t)\right\rangle
$$

and,

$$
\frac{\partial T_{10}\left(x ;\left|\Omega_{0}(t)\right\rangle\right)}{\partial t}=\frac{\partial T_{00 R}\left(x ;\left|\Omega_{0}(t)\right\rangle\right)}{\partial x}-\frac{1}{2} \lambda(t) V_{0}(x) \frac{\partial}{\partial x}\left\langle\Omega_{0}(t)|\hat{\varphi}(x) \cdot \hat{\varphi}(x)| \Omega_{0}(t)\right\rangle
$$

From this we obtain,

$$
\begin{array}{r}
\frac{\partial^{2} T_{00 R}\left(x ;\left|\Omega_{0}(t)\right\rangle\right)}{\partial t^{2}}-\frac{\partial^{2} T_{00 R}\left(x ;\left|\Omega_{0}(t)\right\rangle\right)}{\partial x^{2}}=-\frac{\partial}{\partial t}\left(\lambda(t) V_{0}(x) \frac{\partial}{\partial t}\left\langle\Omega_{0}(t)|\hat{\varphi}(x) \cdot \hat{\varphi}(x)| \Omega_{0}(t)\right\rangle\right) \\
-\frac{\partial}{\partial x}\left(\lambda(t) V_{0}(x) \frac{\partial}{\partial x}\left\langle\Omega_{0}(t)|\hat{\varphi}(x) \cdot \hat{\varphi}(x)| \Omega_{0}(t)\right\rangle\right)
\end{array}
$$

Next consider the situation in Region A (where $x<-L / 2$ ). Since $V_{0}(x)$ is zero throughout this region. we obtain, for Region A,

$$
\frac{\partial^{2} T_{00 R}\left(x ;\left|\Omega_{0}(t)\right\rangle\right)}{\partial t^{2}}-\frac{\partial^{2} T_{00 R}\left(x ;\left|\Omega_{0}(t)\right\rangle\right)}{\partial x^{2}} \underset{x<-L / 2}{=} 0
$$

As long as $L / 2>>t$ we don't have to consider the contribution from Region B. Therefore we will use (5.18) to determine the time evolution of the system in Region A for all times $t$ where $L / 2>>t$. Recall that we have set $L \rightarrow \infty$. The solution to (5.18) is,

$$
T_{00 R}\left(x ;\left|\Omega_{0}(t)\right\rangle\right)=T(x-t)+T(x+t)
$$

where the function $T(x \pm t)$ is determined from the initial conditions at $t=0$. The initial conditions at $t=0$ are,

$$
T_{00 R}\left(x ;\left|\Omega_{0}(0)\right\rangle\right)=T_{00 R}\left(x ;\left|\Omega_{0}\right\rangle\right)
$$

and,

$$
\frac{\partial}{\partial t} T_{00 R}\left(x ;\left|\Omega_{0}(0)\right\rangle\right)=\frac{\partial T_{10}\left(x ;\left|\Omega_{0}(0)\right\rangle\right)}{\partial x}
$$

where $T_{00 R}\left(x ;\left|\Omega_{0}\right\rangle\right)$ is given by (4.14) and (4.15). Also, we have, 


$$
T_{10}\left(x ;\left|\Omega_{0}(0)\right\rangle\right)=T_{10}\left(x ; \hat{P}_{L}\left|0_{\lambda}\right\rangle\right)=T_{10}\left(x+L ;\left|0_{\lambda}\right\rangle\right)=0
$$

where we have used Eq. (5.9) to obtain the final result. Therefore, at $t=0$,

$$
\frac{\partial}{\partial t} T_{00 R}\left(x ;\left|\Omega_{0}(0)\right\rangle\right)=0
$$

Using this we find, in Region A, that,

$$
T_{00 R}\left(x ;\left|\Omega_{0}(t)\right\rangle\right)=\frac{1}{2}\left(T_{00 R}\left(x-t ;\left|\Omega_{0}\right\rangle\right)+T_{00 R}\left(x+t ;\left|\Omega_{0}\right\rangle\right)\right)
$$

According to this result there are two pulses of negative kinetic energy that originate in the region $-a-L<x<a-L$ at $t=0$. One pulse moves in the positive $\mathrm{x}$-direction and the other pulse moves in the negative $\mathrm{x}$-direction. Both pulses are isolated. That is, they is they are separated from any positive kinetic energy density that may exist in Region B by the distance $L / 2$ where $L \rightarrow \infty$.

At time $t=t_{1}$ the kinetic energy density that was confined to the region $|x+L|<a$ at time $t=0$ has now, at $t=t_{1}$, spread out and is contained within the region $|x+L|<a+t_{1}$. The average kinetic energy density within this region is given by,

$$
T_{00, A v e}\left(\left|\Omega_{0}\left(t_{1}\right)\right\rangle\right)=-\frac{\left|E_{K E}\right|}{2\left(a+t_{1}\right)} \text { for }|x+L|<a+t_{1}
$$

The kinetic energy density outside of this region is zero, within the region A. Therefore the spatial quantum inequality is violated.

\section{Violation of the Quantum Interest Conjecture}

Based on the results of the last section it is also evident that the quantum interest conjecture is violated. According to the quantum interest conjecture (Ford et al., 1999; Pretorius et al., 2000; Fewter et al., 2000; Teo et al., 2002; Abreu et al., 2009) any pulse of negative energy must be associated with a larger pulse of positive energy which follows or precedes it. These pulses must be close enough so that any exotic effects due to the negative energy pulse can be compensated for by the associated positive energy pulse. For example if a negative energy pulse fell into a black hole the entropy of the black hole would decrease causing a violation of the second law of thermodynamics. However if a larger positive energy pulse immediately followed the negative energy pulse into the black hole then this violation of the second law would only occur for short enough time to be consistent with the uncertainty principle.

In Region A we have two negative kinetic energy pulses that are moving in opposite directions at the speed of light. With $L \rightarrow \infty$ these pulses are an infinite distance from any positive kinetic energy density that might exist in Region B. Therefore the quantum interest conjecture fails.

\section{Summary and Conclusion}

It has been shown that we can formulate a quantum state that violates the spatial quantum inequality. We start by determining the renormalized kinetic energy density for the state $\left|0_{\lambda}\right\rangle$ in the presence of an inverse square well scalar potential. We show that this state includes a region of negative kinetic energy density. This would result in a violation of the spatial quantum inequality except for the fact that the scalar potential is non-zero. Next we act on the state $\left|0_{\lambda}\right\rangle$ with the translation operator $\hat{P}_{L}$ to produce the state $\left|\Omega_{0}\right\rangle=\hat{P}_{L}\left|0_{\lambda}\right\rangle$. The effect of this is to move the region of negative kinetic energy in the negative x-direction by the amount $L$. If we let $L \rightarrow \infty$ then this region of negative energy density is an infinite distance away from the non-zero scalar potential so that the removal of the scalar potential cannot affect the time evolution of the system in the region of the negative kinetic energy density. This is easily shown to lead to a violation of the spatial quantum inequality.

It is also shown that when the time evolution is considered there will be two pulses of negative kinetic energy density moving in opposite directions at the speed of light. This will result in a violation of the quantum interest conjecture.

\section{References}

Abreu G., \& Visser M. (2009). Quantum Interest in (3+1) dimensional Minkowski space. Phys. Rev. D, Vol. 79 , 065004. http://dx.doi.org/10.1103/PhysRevD.79.065004 
Bostelmann H., \& Fewster C.J. (2009). Quantum Inequalities from Operator Product Expansions. Commun. Math. Phys. 292, 761-795. (arXiv:0812.4760)

Epstein H., Glaser V., \& Jaffe A. (1965). Nonpositivity of the energy density in quantized field theories, Nuovo Cim., Vol. 36, 1016-1022. http://dx.doi.org/10.1007/BF02749799

Eveson, S. P., \& Fewster C. J. (2007). Mass Dependence of Quantum Energy Inequality Bounds. J. Math. Phys., Vol. 48, 093506. (arXiv:math-ph/0702074) http://dx.doi.org/10.1063/1.2779137

Fewster C. J., \& Eveson S. P. (1998). Bounds on negative energy densities in flat spacetime, Phys. Rev. D, Vol. 58, 084010, 1998. (arXiv:gr-qc/9805024v2)

Fewster C. J., \& Teo E. (2000). Quantum inequalities and 'quantum interest' as eigenvalue problems, Phys. Rev. D, Vol. 61, 084012. http://dx.doi.org/10.1103/PhysRevD.61.084012

Fewster C. J. (2005). Energy Inequalities in Quantum Field Theory. (arXiv:math-ph/0501073)

Fewster C. J., \& Osterbrink L.W. (2008). Quantum Energy Inequalities for the Non-Minimally Coupled Scalar Field. J. Phys. A, Vol. 41, 025402. (arXiv:0708.2450)

Flanagan E. E. (1997). Quantum inequalities in two dimensional Minkowski spacetime. Phys. Rev. D, Vol. 55, 2082-2089. (arXiv:gr-qc/9706006v2)

Ford L. H. (1978). Quantum coherence effects and the second law of thermodynamics. Proc. R. Soc. Lond. A, Vol. 364, 227-236. http://dx.doi.org/10.1098/rspa.1978.0197

Ford L.H., \& Roman T.A. (1997). Restrictions on Negative Energy Density in Flat Spacetime, Phys. Rev. D, Vol. 55, 2082-2089. (arXiv:gr-qc/9607003v2)

Ford L. H., Pfenning, M. J., \& Roman T. A. (1998). Quantum Inequalities and Singular Energy Densities. Phys. Rev. D, Vol. 57, 4839-4846. (arXiv:gr-qc/9711030) http://dx.doi.org/10.1103/PhysRevD.57.4839

Ford L.H., \& Roman T. A. (1999). The quantum interest conjecture. Phys. Rev. D, Vol. 60, 104018. http://dx.doi.org/10.1103/PhysRevD.60.104018

Ford L. H. (2010). Negative Energy Densities in Quantum Field Theory. Int. J. Mod. Phys Vol. A25, 2355-2363. (arXiv:0911.3597v1)

Graham N., \& Olum K. D. (2003). Negative energy densities in quantum field theory with a background potential. Phys. Rev. D, Vol. 67, 085014; Erratum-ibid. D69 (2004) 109901 (arXiv:hep-th/0211244v2)

Greiner W., \& Reinhardt J. (1992). Quantum Electrodynamics. Springer-Verlag, Berlin.

Mamaev S. G., \& Trunov N. N. (1981). Vacuum Expectation Values of the Energy-Momentum Tensor of Quantized Fields on Manifolds of Different Topology and Geometry. IV. Russian Physics Journal, Vol. 24, No. 2, 171-174.

Morris M., Thorne K., \& Yurtsever U. (1988). Wormholes, Time Machines, and the Weak Energy Condition. Phys. Rev. Lett., Vol 61, 1446-1449. http://dx.doi.org/10.1103/PhysRevLett.61.1446

Mostepanenko V.M., \& Trunov N. N. (1997). The Casimir Effect and its Applications. Oxford University Press, Oxford.

Pfenning M.J., \& Ford L. H. (1998). Scalar Field Quantum Inequalities in Static Spacetimes. Phys. Rev. D, Vol.57, 3489-3502. (arXiv:gr-qc/9710055) http://dx.doi.org/10.1103/PhysRevD.57.3489

Pretorius F. (2000). Quantum interest for scalar fields in Minkowski spacetime. Phys. Rev. D, Vol. 61, 064005 http://dx.doi.org/10.1103/PhysRevD.61.064005

Solomon D. (2010). A counter-example to the quantum interest conjecture. (arXiv:1007.3258v3)

Solomon D. (2011A). An example of a violation of the quantum inequalities for a massless scalar field in 1-1 dimensional space-time. Adv. Stud. Theor. Phys., Vol. 5, 227-251 (arXiv:1003.1526v6)

Solomon D. (2011B). An example of a violation of the spatial quantum inequality with a comment on the quantum interest conjecture. (arXiv:1006.1637v4)

Solomon D. (2011C). Some ambiguities with point split regularization and its impact on a proof of the spatial quantum inequality. (arXiv:1103.2399v1)

Solomon D. (2011D). Comparing two methods of regularization of the kinetic energy density. (arXiv:1107.5036v2)

Teo E., \& Wong K. F. (2002). Quantum interest in two dimensions, Phys.Rev. D, Vol. 66, 064007. http://dx.doi.org/10.1103/PhysRevD.66.064007 